\title{
Hybride Geschäftsmodelle von Social Enterprises im Spannungsfeld zwischen Lehre und Praxis
}

\author{
Am Markt entwickelte, hybride Geschäftsmodelle von Sozialunternehmen \\ helfen in hohem Maße, die strategische Finanzierungslücke (sog. Tal des \\ Todes) in einer frühen Wachstumsphase durch Ausschöpfen eines breiten \\ Spektrums von Anschlussfinanzierungsmöglichkeiten zu überwinden. Im \\ Vergleich zu den dokumentierten Praxiserfahrungen eines Finanzinterme- \\ diärs scheinen diese Erkenntnisse bisher in den Lehrmedien für Social \\ Entrepreneurship nur unterrepräsentiert zu sein.
}

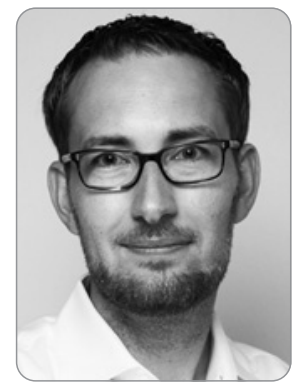

\section{Dr. Markus Freiburg}

ist Gründer und Geschäftsführer der Finanzierungsagentur für Social Entrepreneurship $\mathrm{GmbH}$ (FASE). Als anerkannter Experte für Social Finance und als Mitglied in der Expert Group on Social Entrepreneurship (GECES) der EU Kommission sowie im German National Advisory Board der G7 Social Impact Investing Taskforce ist er ein langjähriger Kenner und Gestalter der Social Entrepreneurship Szene. Er ist Mitglied der Forschungsgruppe Social Entrepreneurship und Social Finance an der Universität Witten/Herdecke.

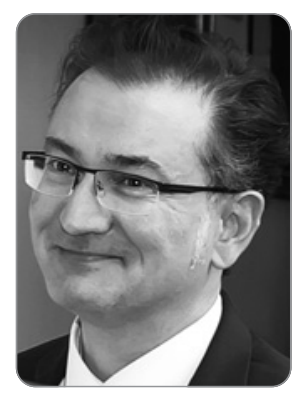

\section{Prof. Dr. Wolfgang Gehra}

hat als Diplom-Kaufmann 20 Jahre Managementerfahrung sowohl in Profit- als auch in Nonprofit-Organisationen. Als Dozent an der Social Entrepreneurship Akademie und an der Hochschule München kennt er aus zahlreichen (Social) Entrepreneurship-Lehrformaten den Entstehungsprozess vieler Geschäftsideen von Anfang an. Bevorzugte Forschungsgebiete: Social Entrepreneurship, Intrapreneurship und soziale Innovationen.

Stichwörter: Social Entrepreneurship, Social Entrepreneurship Education, Hybride Geschäftsmodelle, Business Model, Impact Investing

\section{Ausgangslage von Social Entrepreneuren}

"Sozialunternehmer gewinnen an Bedeutung" (Metzger, 2019, S. 1), die Anzahl an Social Entrepreneuren wächst (vgl. Tete et al., 2018, S. 3), gleichzeitig klagen im aktuellen Social Entrepreneurship Monitor 2018 (ebd. S. 9) über $65 \%$ der befragten Social Entrepreneure über die Anschlussfinanzierung als wesentliche Hürde, um ihre Orga- nisation weiter zu entwickeln bzw. um das Überleben $\mathrm{zu}$ sichern.

Soziale Innovationen, initiiert von Social Entrepreneuren, gelten aber als wichtiger Baustein zur Lösung gesellschaftlicher Herausforderungen und sind expliziter Bestandteil der Hightech Strategie 2025 der Bundesregierung (vgl. $B M B F$, S. 4, 10, insbes. 48). Wenn soziale Innovationen breit umgesetzt werden, können sie Systeme nachhaltig verändern und Einsparpotentiale in Milliardenhöhe mit sich bringen (vgl. Ashoka, McKinsey, 2019, S. 4 ff.).

Sozialunternehmen werden hier verstanden als Organisationen, die auf unternehmerische, innovative Art und Weise zur Lösung sozialer und ökologischer Probleme beitragen und dabei ein auf ihren Leistungen basierendes Einkommen generieren (vgl. Scheuerle et al., 2013, S. 4).

Ziel des vorliegenden Beitrags ist es deshalb, mit Blick auf die eingangs geschilderte Herausforderung der überlebenssichernden Anschlussfinanzierung, am Markt entwickelte Geschäftsmodelle zwischen Gemeinnützigkeit und Gewerblichkeit anhand von zwei Fallbeispielen vorzustellen und die Berücksichtigung solcher Modelle in der Lehre zu Social Entrepreneurship zu beleuchten.

2. FASE als Brückenbauer zwischen Sozialunternehmen und wirkungsorientierten Investoren

Die Finanzierungsagentur für Social Entrepreneurship GmbH (FASE) unterstützt als Intermediär seit Anfang 2013 Sozialunternehmen beim Einwerben von hybridem Wachstumskapital bei wirkungsorientierten Investoren. Damit will $F A$ SE Sozialunternehmen helfen, ihre strategische Finanzierungslücke („Tal des Todes“) zu überbrücken und damit eines der drängendsten Hindernisse für die Verbreitung 
Quelle: FASE, 2014, S. 14.

Abb. 1: Hybride Gesellschaftsstruktur von Discovering Hands
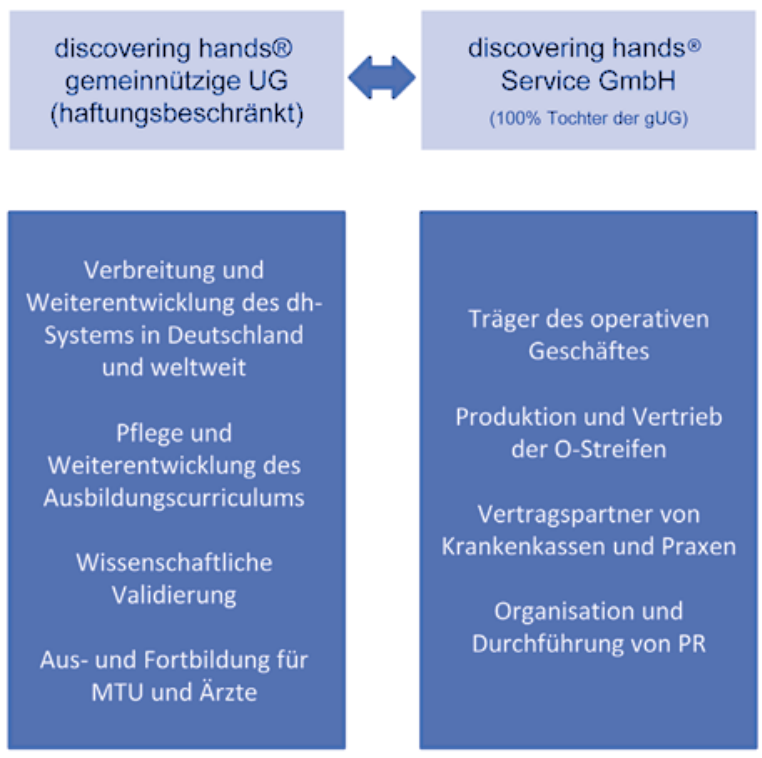
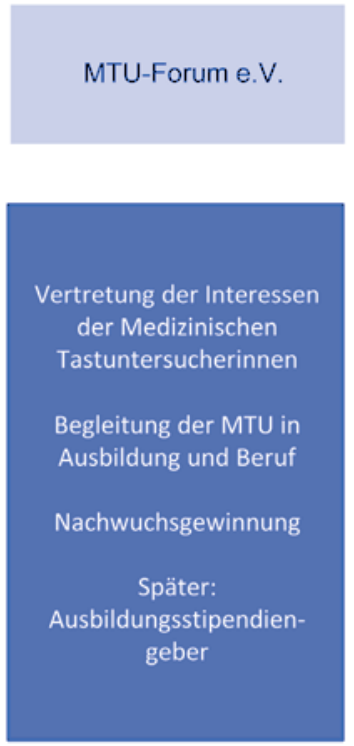

von sozialen Innovationen in Deutschland beseitigen und einen Beitrag zur Transformation des Finanzierungsmarktes leisten (vgl. Freiburg/Moehrle, 2017, dies., 2018a, dies., 2018b).

FASE, initiiert von Ashoka - der weltweit größten Organisation zur Unterstützung von sozialem Unternehmertum - , versteht sich als Brückenbauer zwischen Sozialunternehmen und Investoren, um durch Weiterentwicklung des Geschäftsmodells zur Finanzierungsreife und um durch professionelle Transaktionsunterstützung beim Einwerben von Wachstumskapital genau die Unterstützung zur Anschlussfinanzierung $\mathrm{zu}$ bieten, die einleitend als wesentliches Hindernis benannt wurde.

Dabei geht es um Finanzierungen im Bereich zwischen $100 \mathrm{~T} €$ und 1 Mio. $€$ in einer frühen Wachstumsphase, die sich an die meist durch Spenden, Preise und Eigenmittel finanzierte Experimentier- bzw. Startup-Phase anschließt. Ausgesuchte Sozialunternehmen mit sozial innovativen und skalierbaren Geschäftsmodellen werden in einem mehrstufigen Pipeline-Prozess hinsichtlich verschiedener Kriterien wie Social Impact, Geschäftsmodell oder Team begutachtet.

\section{Dokumentierte Erfahrungen aus der Praxis von FASE}

Nach mittlerweile über sechsjähriger Tätigkeit konnte durch das FASE-Team folgende empirische Erfahrung dokumentiert werden:

Über 550 Sozialunternehmen, auf der Suche nach Wachstumsfinanzierung, wurden begutachtet. Mehr als 40 davon wurden in verschiedenen Finanzierungsrunden bis Ende 2018 beim Einwerben eines Gesamtvolumens in Höhe von mehr als 20 Mio. $€$ erfolgreich unterstützt.

Von diesen mehr als 40 erfolgreich finanzierten Sozialunternehmen haben etwa die Hälfte ein hybrides Geschäfts- modell, d.h. gewerbliche- und gemeinnützige Elemente werden kombiniert. Diese hybriden Elemente im Geschäftsmodell spiegeln sich weiterhin in der Struktur der Organisation, der Rechtsform und letztendlich der Ausgestaltung der Finanzierung. Rückzahlbare Finanzierungsformen eignen sich, in profitorientierte Teile der Organisation zu investieren und gleichzeitig unterstützen Spenden und/oder öffentliche Fördergelder den Non-Profit-Teil. Mit diesem Ansatz gelingt es FASE, das breite Spektrum von unterschiedlichen Motiven der Investoren, von reiner Spendenorientierung bis hin zu Renditeerwartungen auf Kapitalmarktniveau, abzudecken und dadurch Finanzierungen in dieser schwierigen Wachstumsphase zu ermöglichen und sicher zu stellen (vgl. FASE, 2015, S. 7 und 46, sowie FASE, 2018, S. 14).

\section{Fallbeispiele für hybride Geschäftsmodelle und Finanzierung}

An zwei von FASE betreuten und mit hybriden Finanzierungen ausgestatteten Sozialunternehmen lässt sich das Zusammenwirken von Geschäftsmodell, Organisation und Rechtsform, jeweils in kombinierter Form von Profit- und Non-Profit-Elementen, besonders gut verdeutlichen. Dies sind Discovering Hands und Team U.

\subsection{Discovering Hands}

Discovering Hands bildet blinde Frauen zu Medizinischen Tastuntersucherinnen (MTU) nach einem standardisierten Tastverfahren aus. Deren überdurchschnittlicher Tastsinn optimiert die Früherkennung von Brustkrebs und erhöht signifikant die Überlebenschancen von Patientinnen. Gleichzeitig erleben die zur MTU qualifizierten Frauen ihre Blind- 

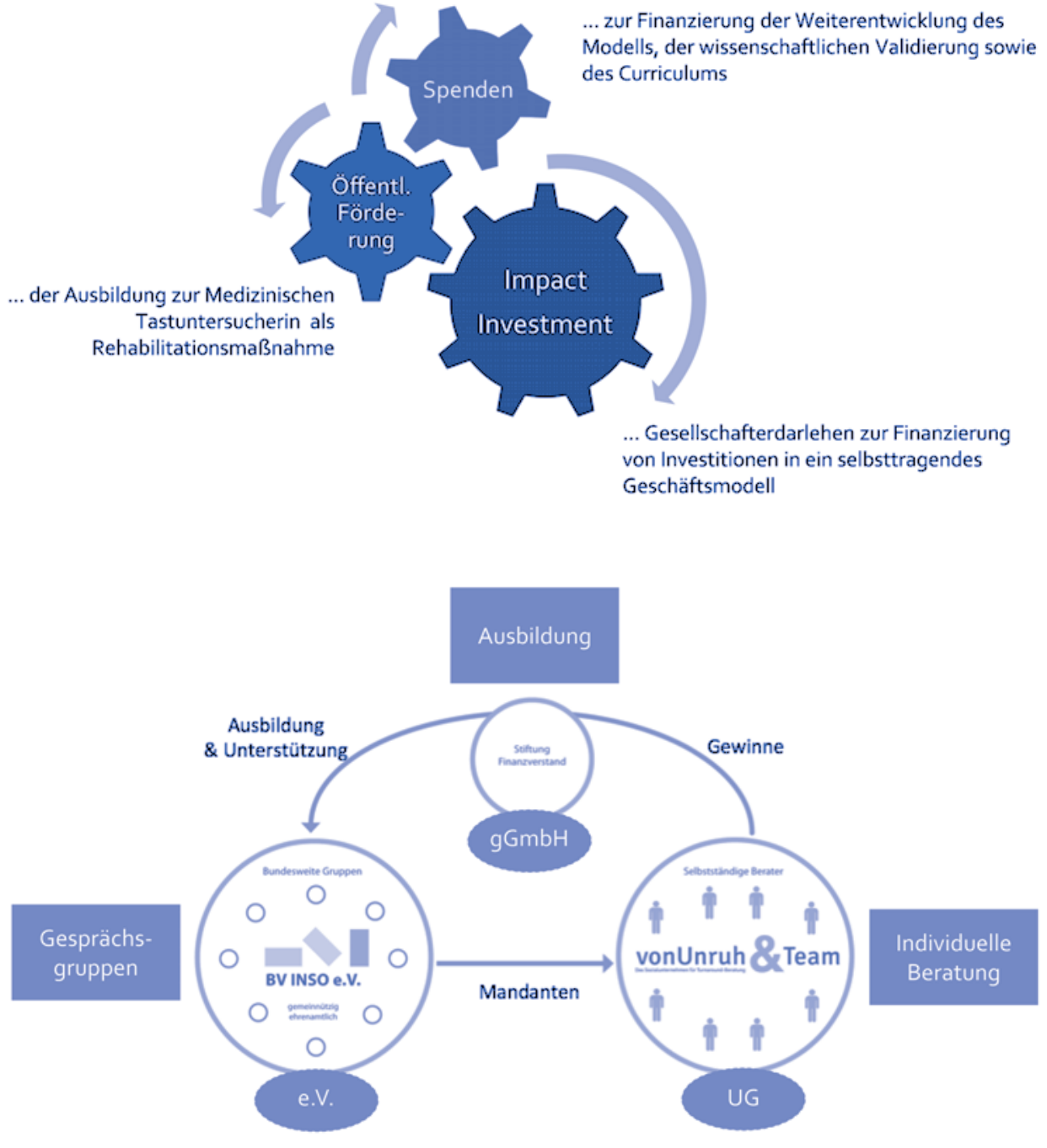

Quelle: FASE, 2014, S. 16.

Abb. 2: Hybride Finanzierungsstruktur von Discovering Hands
Quelle: FASE, 2013, S. 8.

Abb. 3: Hybrides Geschäftsmodell von Team U heit nicht als Behinderung, sondern als wertvolle Ressource, die ihnen zur Inklusion in die Arbeitswelt hilft (vgl. FASE, 2014, S. 7 ff.). Die hybride Gestaltung der Rechtsform und der Organisation vollzieht sich in Form von zwei gemeinnützig ausgerichteten Körperschaften und einer gewinnorientierten $\mathrm{GmbH}$ (vgl. Abb. 1).

Die Gewinne der rein marktwirtschaftlich geführten Discovering Hands Service GmbH aus dem Vertrieb der Orientierungsstreifen und den Erstattungen durch Krankenkassen und Arztpraxen fließen zurück in die Muttergesellschaft, der als gemeinnützige UG gegründete Discovering Hands gUG oder werden in der $\mathrm{GmbH}$ für weitere Investitionen thesauriert. Die Ausschüttungen der $\mathrm{GmbH}$ zugunsten der gemeinnützigen gUG erzeugen einen in sich geschlossenen Finanzierungskreislauf und verhindern gleichzeitig mögliche Konflikte mit philanthropisch motivierten Geldgebern. Der Verein MTU-Forum e. V. , ebenfalls als gemeinnützig anerkannt, fungiert als Berufsverband für die Tastuntersucherinnen und begleitet diese in Ausbildung und Beruf (vgl. FASE, 2014, S. 14 und 15). Abb. 2 verdeutlicht die unterschiedlichen Finanzierungsquellen und das Ineinandergreifen der Finanzierungsarten.
Die hybride Ausgestaltung des Geschäftsmodells und der rechtlichen Organisation ist Voraussetzung für eine eben solch hybride Finanzierungsstruktur. Philanthropisch orientierte Spender, beispielsweise private Geldgeber oder Stiftungen fördern die als gemeinnützig anerkannten Körperschaften Discovering Hands gUG und MTU Verein e. V. Öffentliche Geldgeber in Form der Träger von Reha-Maßnahmen finanzieren die Ausbildung zur MTU. Impact- und finanzorientierte Investoren finanzieren über verzinste $\mathrm{Ge}$ sellschafterdarlehen sowohl die gUG als auch die $\mathrm{GmbH}$ (vgl. FASE, 2014, S. 15 ff.).

\subsection{Team $U$}

Das Sozialunternehmen von Team U bietet als Beratungsgesellschaft Krisen- und Tournaround-Beratung für UnternehmerInnen an (vgl. $A b b .3$ ). Auch in diesem Fall konnte mit Hilfe von FASE eine maßgeschneiderte Finanzierung entwickelt werden, die in Erweiterung des erstgenannten Beispiels an dieser Stelle besondere Erwähnung finden soll. Während sich der Verein über Spenden bzw. Mitgliedsbeiträge und die $\mathrm{gGmbH}$ über Fundraising finanziert, wurde für 


\begin{tabular}{|c|c|c|c|c|}
\hline \multicolumn{5}{|c|}{ Nennung hybrider Geschäftsmodelle in Lehr- und Infomaterialien zu Social Entrepreneurship } \\
\hline Titel: Praxisratgeber / Lehrbuch & Verlag / Herausgeber / Autor & Jahr & $\begin{array}{l}\text { Nennung / Erläuterung } \\
\text { hybrider Geschäfts- } \\
\text { modelle }\end{array}$ & $\begin{array}{l}\text { Hybride } \\
\text { Rechts- } \\
\text { formen }\end{array}$ \\
\hline Praxisleitfaden Soziales Unternehmertum & $\begin{array}{l}\text { Bundesministerium für Wirtschaft und } \\
\text { Energie }\end{array}$ & 2018 & nein & nein \\
\hline Social Startup Guide & $\begin{array}{l}\text { start-up center der OTH Regensburg und } \\
\text { enactus Regensburg e.V. }\end{array}$ & o.J. & nein & nein \\
\hline $\begin{array}{l}\text { Deutscher Social Entrepreneurship } \\
\text { Monitor } 2018\end{array}$ & $\begin{array}{l}\text { Social Entrepreneurship Netzwerk } \\
\text { Deutschland e.V. (SEND) }\end{array}$ & 2018 & ja & ja \\
\hline $\begin{array}{l}\text { Entrepreneurship (Lehrbuch), Kap. } 18 \\
\text { Social Entrepreneurship }\end{array}$ & Grichnik, Brettel, Koropp, Mauer & 2017 & ja S. 437 & nein \\
\hline Kursbuch Wirkung & phineo $g A G$ & 2018 & nein & nein \\
\hline Lehrbuch Entrepreneurship & Fueglistaller et al. & 2015 & $\begin{array}{l}\text { im Kapitel SE nicht } \\
\text { explizit }\end{array}$ & nein \\
\hline $\begin{array}{l}\text { Mit Mehrwert überzeugen - der Social } \\
\text { Businessplan für Sozialunternehmen }\end{array}$ & Klaus Schellberg (Hrsg.) & 2018 & $\begin{array}{l}\text { Nennung im Sinne von } \\
\text { Quersubventionierung } \\
\text { S. } 33\end{array}$ & nein \\
\hline Social Entrepreneurship & Constant Beugré & 2017 & $\begin{array}{l}\text { Nennung als hybrid } \\
\text { social venture S. } 5\end{array}$ & nein \\
\hline $\begin{array}{l}\text { Munich Business School Burkett, I. } \\
\text { (2016). Using the Business Model Canvas } \\
\text { for Social Enterprise Design. Foliensatz }\end{array}$ & $\begin{array}{l}\text { https://mbs.edu/getmedia/91cc0d01-3641- } \\
\text { 4844-b34c-7aee15c8edaf/Business-Model- } \\
\text { for-SE-\%20\%20Design-Burkett.pdf }\end{array}$ & 2018 & nein & nein \\
\hline \multicolumn{5}{|l|}{ MOOC } \\
\hline $\begin{array}{l}\text { SEA:MOOC Enabling Entrepreneurs to } \\
\text { Shape a Better World }\end{array}$ & SEA Akademie & 2018 & $\begin{array}{l}\text { nein Osterwalder } \\
\text { Business Models Part } \\
1-3\end{array}$ & nein \\
\hline $\begin{array}{l}\text { Changemaker MOOC Social } \\
\text { Entrepreneurship }\end{array}$ & iversity Springer Nature & $\begin{array}{l}\text { Abruf am } \\
2.5 .2019\end{array}$ & nein & nein \\
\hline $\begin{array}{l}\text { Social Innovation MOOC (EBS, World } \\
\text { Vision Stiftung) }\end{array}$ & iversity Springer Nature & $\begin{array}{l}\text { Abruf am } \\
2.5 .2019\end{array}$ & nein & nein \\
\hline $\begin{array}{l}\text { New Business Models - Working } \\
\text { Together on Value Creation MOOC }\end{array}$ & Jan Jonker, iversity Springer Nature & $\begin{array}{l}\text { Abruf am } \\
2.5 .2019\end{array}$ & nein & nein \\
\hline
\end{tabular}

Tab. 1: Nennung hybrider Geschäftsmodelle in Lehr- und Infomaterialien zu Social Entrepreneurship.

die Beratungsgesellschaft in Form einer GmbH eine Finanzierungslösung erdacht, die Wachstum und die entsprechende Skalierung der sozialunternehmerischen Idee ermöglicht. Eine Mezzanine-Finanzierung, ausgestaltet als Genussrechtskapital ohne Verlustbeteiligung bildet die Basis des Finanzierungsmodells und erhöht damit das wirtschaftliche Eigenkapital. Eine variable Vergütung in prozentualer Abhängigkeit vom Umsatz bei gleichzeitiger nominaler Begrenzung auf einen Höchstbetrag, in Verbindung mit einer Ausgleichszahlung am Ende der Laufzeit, berücksichtigt die Interessen von Investoren nach erfolgsabhängiger Rendite für das übernommene unternehmerische Risiko. Die Bedürfnisse des Sozialunternehmens werden durch dieses Finanzierungsmodell neben der grundsätzlichen Möglichkeit an Wachstumskapital zu gelangen, vor allem durch die Flexibilität hinsichtlich Erfolgsabhängigkeit und Tilgung abgebildet (vgl. FASE, 2013, S. 12 ff.).

\section{Folgerungen für die Social Entrepreneurship Lehre}

Ein Blick in die in der Hochschullehre verwendete Lektüre, in die M00Cs (Massive Open Online Courses) und in die Praxisratgeber, die auf Social Entrepreneurship-Portalen häufige Erwähnung finden (z.B. www.gruenderkueche.de), zeigt, ohne Anspruch auf Vollständigkeit, das in Tab. 1 enthaltene Bild.

Von neun aktuellen Lehrbüchern bzw. Praxisratgebern aus den Jahren 2017 und 2018 zum Thema Social Entrepreneurship erwähnen nur zwei Quellen das Thema "hybride Geschäftsmodelle“ explizit und zwei im Sinne von "Quersubventionierung“ bzw. als "hybrid social venture". In den vier betrachteten M00Cs werden hybride Geschäftsmodelle gar nicht thematisiert. Von den insgesamt analysierten 13 Medien erwähnt nur ein Autorenteam die Möglichkeit hybrider Rechtsformgestaltung.

Im Gegensatz zur festgestellten Relevanz in der Finanzierungspraxis am Markt, spielen hybride Betrachtungsweisen in Bezug auf Geschäftsmodell, Organisation und Rechtsform in aktuellen Medien der Social Entrepreneurship Lehre scheinbar eine nur untergeordnete Rolle. Die klassische Einteilung in profitorientierte Geschäftsmodelle auf der einen und sozial orientierten Modellen auf der anderen Seite scheint angesichts der dargestellten, erweiterten Finanzierungsmöglichkeiten für hybrid orientierte Sozialunternehmen verkürzt zu sein.

Ein allgemeines Modell für eine hybride Gestaltung eines Sozialunternehmens, sozusagen als Anregung für die Lehre, könnte demnach wie in $A b b .4$ dargestellt aussehen. 


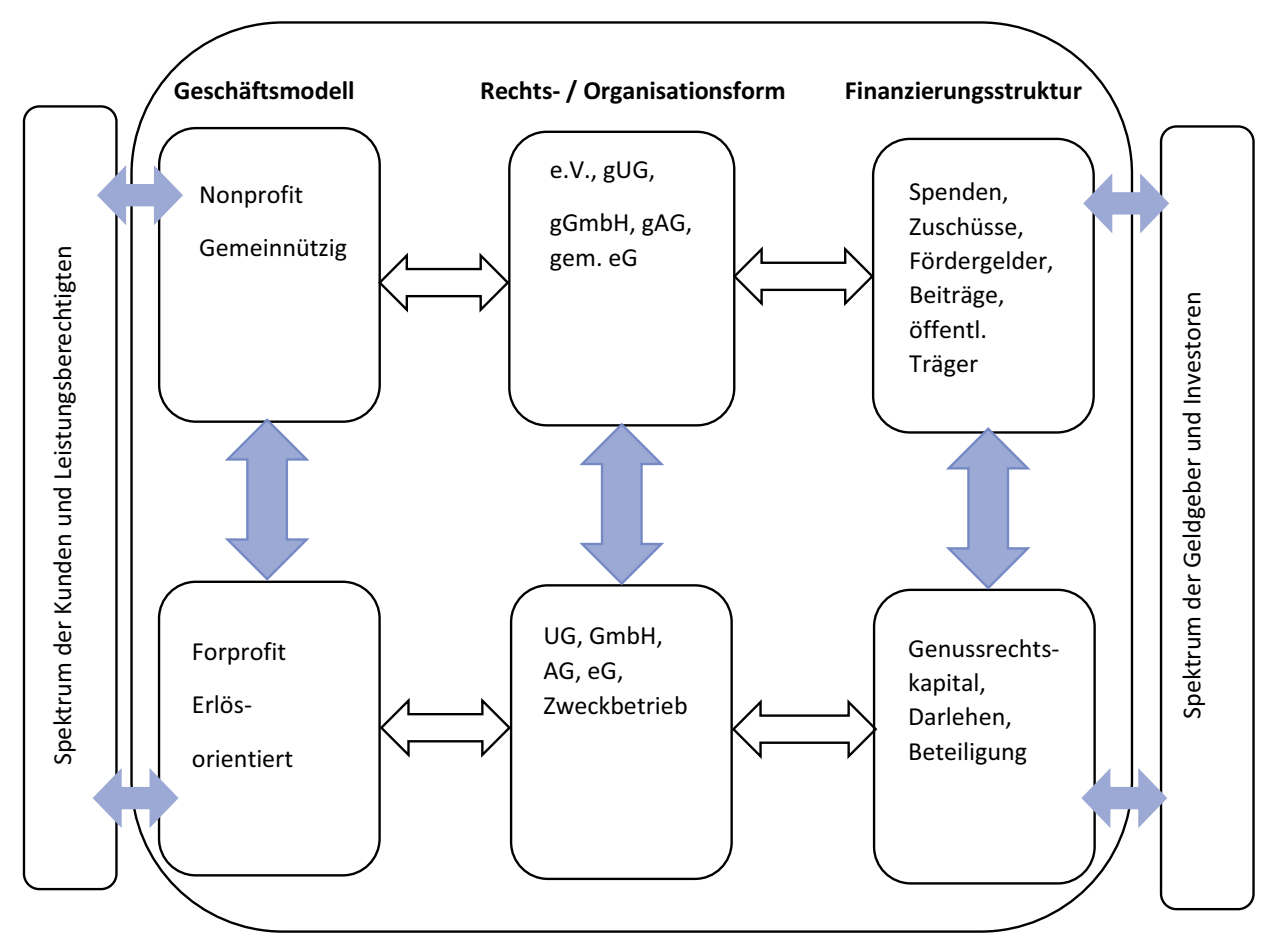

Abb. 4: Hybride Gestaltung eines Sozialunternehmens.
Im Sinne einer praxisorientierten Verbindung der Social Entrepreneurship-Lehre mit Finanzintermediären und Investoren wäre daher die Schlussfolgerung, bereits bei der Geschäftsmodellentwicklung gewerblich und gemeinnützig zu denken, um die Dichotomie zwischen profit- und sozialorientiertem Unternehmertum aufzubrechen.

\section{Ausblick}

Momentan geben ca. 12 \% der im Rahmen des Deutschen Social Entrepreneurship Monitors 2018 befragten Organisationen an, über zwei Rechtsformen zu verfügen. Beispielsweise in Form einer $\mathrm{GmbH}$ in Kombination mit einem eingetragenen, gemeinnützigen Verein oder einer gGmbH (vgl. Tete et al., 2018, S. 24 und SEFORIS, 2016, S. 15).

Demzufolge wäre es, auch aus Sicht eine Finanzintermediärs wie FASE, interessant zu beobachten, inwieweit sich die Anzahl, Qualität und Vermittelbarkeit von Sozialunternehmen zu Investoren verbessert, wenn bereits in den Anfangsstadien der Geschäftsmodellentwicklung, häufig schon in Lehre und Studium, systematischer die Möglichkeit von hybriden Formen Berücksichtigung fände.

\section{Literatur}

Ashoka Deutschland gGmbH und McKinsey \& Co, Inc., Wenn aus klein systemisch wird - Das Milliardenpotenzial sozialer Innovationen, München 2019.

BMBF Bundesministerium für Bildung und Forschung (Hg.), Hightech Strategie der Bundesregierung 2025 - Forschung und Innovation für die Menschen, Berlin 2018.
FASE, EU II Project Final Report, Facilitating hybrid Finance for Social Enterprises, 2018, URL: https://fa-se.de/static/fa_se_de/uploads/2018/07 /Final-Project-Report-VS-2016-0353.pdf (Abruf am 27.06.2019).

FASE, Social Finance Fallstudie: Finanzierung eines Sozialunternehmens mit hybridem Geschäftsmodell: vonUnruh \& Team, 2013, URL: https://fase.de/static/fa_se_de/uploads/2018/06/131213_Finanzierung_von UnruhTeam_CaseStudy.pdf (Abruf am 20.05.2019).

FASE, Fallstudie Social Entrepreneurship: Skalierung und Finanzierung einer Sozialen Innovation: Discovering Hands, 2014,

URL: https://fa-se.de/static/fa_se_de/uploads/2018/04/141006_Fallst udie-Social-Entrepreneurship_DH26120207.pdf (Abruf am 15.05.2019).

FASE, Final Report EU Project, Creating Collaborative Funding Models for Social Enterprises, 2015, URL: https://www.fa-se.de/static/fa_se_de/up loads/2015/12/FASE-Final-Report-EU-Project-July-2015.pdf (Abruf am 27.06.2019).

Freiburg, M., Moehrle, C., Financing Agency for Social Entrepreneurship (FASE): An intermediary for hybrid financing, Germany, in: $O E C D / E U$ (Hrsg.), Boosting Social Enterprise Development: Good Practice Compendium, Paris 2017, S. 111-119.

Freiburg, M., Moehrle, C., Vom Brückenbau zwischen Social Entrepreneurs und Impact Investors, in: P. Bungard (Hrsg.), CSR und Geschäftsmodelle Auf dem Weg zum zeitgemäßen Wirtschaften, Berlin/Heidelberg 2018a, S. 231-245.

Freiburg, M, Moehrle, C., Building a Thriving Ecosystem for Social Enterprise Finance, in: $K$. Wendt (Hrsg.), Positive Impact Investing - A Sustainable Bridge Between Strategy, Innovation, Change and Learning, Cham 2018b, S. 217-230.

Metzger, M., Social Entrepreneurs in Deutschland: Raus aus der Nische 154.000 "junge" Sozialunternehmer im Jahr 2017, KFW Research 2019, URL: https://www.kfw.de/PDF/Download-Center/Konzernthemen/Resea rch/PDF-Dokumente-Fokus-Volkswirtschaft/Fokus-2019/Fokus-Nr.-238Januar-2019-Sozialunternehmer.pdf (Abrufdatum 3.5.2019).

SEFORIS, Landesbericht Deutschland, Eine erste Analyse und ein erstes Profil von Sozialunternehmen in Deutschland vorgelegt vom SEFORÏS Forschungskonsortium, 2016, URL: https://static1.squarespace.com/ static/56d2eebbb654f9329ddbd20e/t/57e5a4cdbebafba4111f0c6d/ 1474667731430/Country-Report-DE_DE-FINAL.pdf (Abrufdatum 15.07.2019). 
Scheuerle, T., Glänzel, G., Knust, R., Then, V., Social Entrepreneurship in Deutschland. Potentiale und Wachs-tumsproblematiken, Heidelberg/ Frankfurt am Main 2013.

Tete, P., Wunsch, M., Menke, C., Deutscher Social Entrepreneurship Monitor 2018, Social Entrepreneurship Netzwerk Deutschland e. V. (SEND), URL: https://www.send-ev.de/uploads/dsem-2018_web.pdf (Abrufdatum 3.5.2019).

\section{Wichtig für Alle.}

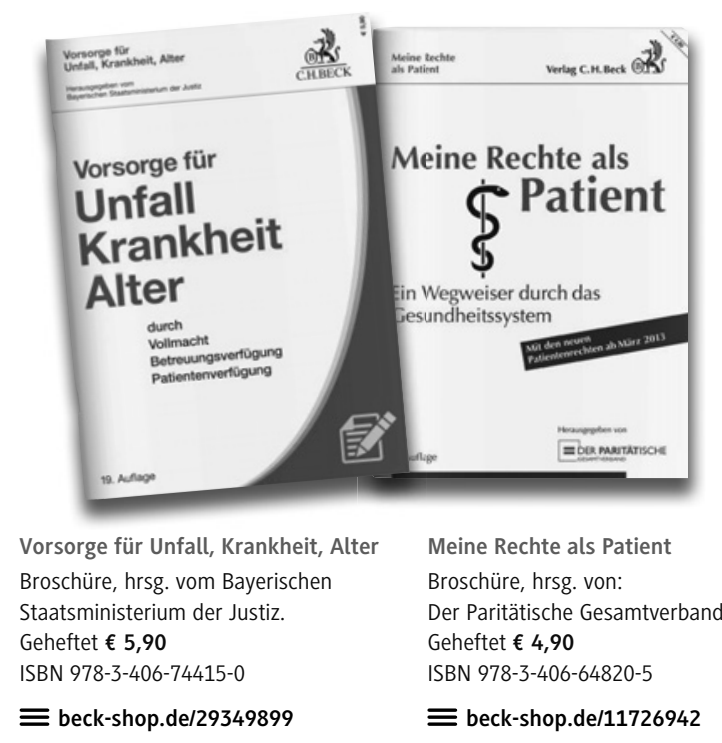

Erhältlich im Buchhandel oder bei: beck-shop.de I Verlag C.H.BECK oHG . 80791 München I kundenservice@beck.de I Preise inkl. MwSt. I 156044

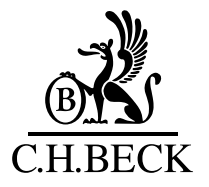

\section{Bezugspreise 2021}

\section{WiSt - Wirtschaftswissenschaftliches Studium}

Inkl. WiSt-Archiv online für einen Arbeitsplatz

In Gemeinschaft mit dem Verlag Franz Vahlen.

Die Bezugspreise (einschließlich MwSt.)

betragen ab 1. Januar 2021:

Normalpreis

halbjährlich € 122,-

Vorzugspreis für Studenten fachbezogener

Studiengänge (gegen Nachweis) halbjährlich € 59,-

Kombipreis inkl. Campuslizenz halbjährlich $€ 185$,-

jeweils zuzüglich Vertriebsgebühren

halbjährlich $€ 8,50$.

Abbestellung bis 6 Wochen vor Halbjahresende.

Einzelheft

$€ 27,-$

Bestellen Sie bei Ihrem Buchhändler oder bei:

Bestellen Sie bei Threm Buchhändler oder bei:
beck-shop.de oder Verlag C.H.BECK · 80791 München · Fax: 089/38189-358 · www.beck.de

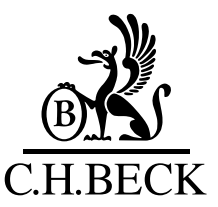

